some detail what could not be explained, due to scientific publication conventions, in our paper.

The numbers of microorganisms recovered from the 22 ventilator tubes treated with disinfectant that had been used 5 or fewer days were as follows: 19 had zero, 1 had 2,1 had 6 , and 1 had 4,000 organisms. In the Materials and Methods section we indicated that the numbers of microorganisms recovered from ventilator tubing would be expressed as means (averages) with $95 \%$ confidence intervals. We chose this method expressing the data for several reasons. First, confidence intervals take into account both population variability and sample size in expressing "confidence" in the population sample estimate $(\overline{\mathrm{X}})$. Second, our other option for presenting the data was to use median values and show ranges. Although a perfectly legitimate method for describing the central tendency of data, I am not sure the readers would have appreciated having numbers of organisms recovered from untreated and washed tubes expressed in means $\pm 95 \%$ confidence intervals and those from disinfected tubes expressed in medians and ranges. Similarly, the use of medians and ranges throughout would have been awkward in tabular form and unless careful notation of the range $(0$ to 4000 in the $\leqslant 5$ day group) was taken the median values for all $6(\leqslant 5$ days through 26 to 30 days, Table 1) groups would have been zero. Such data presentation would have made it appear to the more casual reader that if this disinfectant were used for 30 days, zero organisms would be expected. Certainly, the data do not support that. Finally, means and $95 \%$ confidence intervals are useful in easily comparing populations. Since the confidence intervals of all groups (see above) included zero the reader can easily conclude that no difference was apparent between groups and if repeated samples were taken the probability of a zero result was high.

I am not surprised by the AOAC test results reported in Pfaffenroth and coworkers' letter. After use, most disinfectants become diluted because wet equipment is immersed in the solution. The Environmental Protection Agency which must approve manufacturers' claims does not take this "real world" variable into account in their testing procedure (for further EPA disinfectant problems see Gröschel's editorial, Infection Control, May/June 1983). We emphasized in our paper by way of a special note (p. 243) that routine microbiological monitoring of liquid chemical disinfected respiratory therapy equipment was recommended by the Centers for Disease Control. I am a little disturbed by Pfaffenroth and co-workers' finding that routine monitoring showed efficacy at 30 days but AOAC tests showed only $36 / 60$. Such data should be submitted for publication; if for no other reason than to stress the far from perfect methods we have available to evaluate disinfectants.

Timothy R. Townsend, MD Johns Hopkins Hospital Baltimore, Maryland

\section{Use of Multidose Vials}

To the Editor:

We have received conflicting information regarding the use of multidose vials and have been unable to obtain authoritative sources of information. The question is how long can the contents be considered safe for injection once the diaphragm of the vial has been punctured? Some sources state 30 days from the first puncture and others state until the expiration date for the contents of the vial.

Sharon M. Howard, RN Director of Nursing Ross Care Center Merrillville, Indiana

The preceding letter was referred to Mark Eggleston, PharmD, and John P. Burke, $M D$, for their replies.

Presently, there are conflicting results reported in the literature. Multiple dose vials (MDVs) for parenteral use are potentially a source of nosocomial infections. Similar parenteral products (improperly used IV catheters, contaminated single use IV fluids) have been implicated in sporadic as well as epidemic cases of bacteremia. $^{1}$
Actual clinical infections resulting from contaminated MDVs have not been reported frequently in the medical literature. However, Olsen et $\mathrm{al}^{2}$ documented eight cases of Flavobacterium meningosepticum bacteremia caused by extrinsic contamination of MDVs by poor aseptic technique.

There are several studies and reports that address the possibility of contamination of MDVs during use and the ability of organisms to survive in a variety of medications packaged in MDVs. ${ }^{3-7}$ Most have discovered a low rate of contamination. Highsmith, Allen and Greenhood ${ }^{8}$ showed that the risk of significant microbial contaminacion for some types of medication appears low. They noted, however, that several organisms survived or grew in a MDV containing lidocaine. The lidocaine solution also contained endotoxin after contamination with Pseudomonas cepacia, as did insulin contaminated with enterococcus. Borghaus et $\mathrm{al}^{9}$ reported that if an MDV is contaminated with a particular agent that is resistant to the bacteriostatic agent present, it very quickly may become a potential source of infection to patients. These researchers found that bacteria recovered from unopened vials of the anesthetic fentanyl could be grown in the drug alone and in the preservative, parahydroxybenzoic acid. The generation time was less than four hours.

In contrast, a study conducted at the National Naval Medical Center examined 1,223 samples from 864 vials which had been in use from 1 to 402 days. They could find no contamination in any MDV, and concluded that MDVs may safely be used until empty or until the manufacturer's expiration date, whichever occurs first. ${ }^{10}$

Bawden et al" examined MDVs after collection from hospital nursing units and after deliberate contamination. Bacteria were isolated from deliberately contaminated MDVs when inoculated with 1 to 100 colony forming units $/ \mathrm{ml}$ or greater when the sample was tested within one hour after contamination. Only one vial was positive at 16 hours and none were positive beyond that time. No bacterial contamination was found in the MDVs collected from the nursing stations. The researchers concluded 
that the contamination rate was probably less than 4 per 1,000 .

Although the actual rate and prevalence of contaminated MDVs cannot be determined, several factors should be considered when making recommendations for discarding opened vials: 1) intrinsic contamination of unopened vials-each vial should be inspected for cracks, defective seals, or turbidity before use; 2) frequency and technique with which MDVs are entered-careful aseptic technique should be followed each time the MDV is used. Vials that are so entered and especially those containing expensive medications used frequently in a specific location can probably be used entirely.

MDVs that are carelessly punctured and not reused for an indefinite period should be discarded, eg, some emergency room MDVs or MDVs on cardiac resuscitation carts; 3 ) location of MDVs-operating rooms and intensive care units have patients who are considerably more vulnerable to nosocomial bacteremia; therefore, MDVs in these areas should be discarded relatively soon after initial use, thus reducing the potential for contamination; and 4) the activity of the bacteriostatic agent against various bacteria-a study by Young et $\mathrm{al}^{7}$ showed that growth and replication of certain bacteria is unlikely when they are in prolonged contact with adequate and active bacteriostatic agents found in MDVs.

The final recommendation for control measures is still a matter of judgment. As with other infection control problems, the cost, feasibility, and eventual effectiveness of the measure must be weighed against the benefits to be derived from it.

\section{REFERENCES}

1. Maki DG, Goldmann DA, Rhame FS: Infection control in intravenous therapy. Ann Intern Med 1973; 79:867-887.

2. Olsen H, Frederiksen WC, Siboni K: Flavobacterium meningosepticum in 8 nonfatal cases of postoperative bacteremia. Lancel 1965; 1:1294-1296.

3. Bothe J: Study shows contamination in multiple dose vials. AORN J 1973; 17:111-114.

4. Corley CE, Manos JP, Thomas JD: Multiple dose vials: A source of contamination? JSC Med Assoc 1968; 64:461-464.

5. Kohan S, Carlin H, Whitehead R: A study of contamination of multiple dose medication vials. Hospitals 1962; 36:78-80.
6. Rosenzweig AL: Potential health hazards in the multiple dose vial. Hospitals 1964; 38:71-76.

7. Young JA, Collette TS, Brehm WF: Sterility of multiple dose vials after repeated use. $A m$ Surg 1958; 24:811-814.

8. Highsmith AK, Greenhood GP, Allen JR: Growth of nosocomial pathogens in multiple-dose parenteral medication vials. $J$ Clin Microbiol 1982; 15:1024-1028.

9. Borghaus JGA, Hosli MTC, Olsen $\mathrm{H}$, et al: Pseudomonas cepacia bacteremia due to intrinsic contamination of an anesthetic. Acta Pathol Microbiol Scand 1979; 87:15-20.

10. Multiple dose vials safe, new study discovers. Hospital Infection Control 1982; 9:159.

11. Bawden JC, Jacobson JA, Jackson JC, et al: Sterility and use patterns of multiple-dose vials. Am J Hosp Pharm 1982; 39:294-297.

Mark Eggleston, PharmD Clinical Pharmacist

Epidemiology Department Potomac Hospital Woodbridge, Virginia

There is no specified length of time that a multidose vial (MDV) can be considered safe. Microbial contamination may occur with the first entry, rendering the product unsafe for any further use. Curiously, clinical infections resulting from use of contaminated medications from MDVs appear to be rare. However, instances undoubtedly occur that are unrecognized and unreported. Recently, the potential hazards were realized in two separate outbreaks of group A streptococcal abscesses in infants and children who received diphtheriatetanus-pertussis (DTP) vaccine from MDVs that had been contaminated. ${ }^{1}$ Challenge studies conducted at the Centers for Disease Control (CDC) indicated that a case strain of streptococcus could survive 15 days at $4^{\circ} \mathrm{C}$ in DTP vaccine.

Nonetheless, the CDC Guidelines on Infection Control contain the statement that "unless an expiration date is stated on the product label or package insert, it is not known if multiple-use containers, once entered, should be discarded after a specific or arbitrary length of time." 2 The US Pharmacopeial Convention also has regarded "any time limit put on the use of a MDV after its first opening as strictly arbitrary." 3

The extent of the potential problem of microbial contamination of MDVs has not been fully investigated, and more detailed information is needed for the establishment of safe practices in health-care facilities. Several groups have performed prevalence surveys to determine the rate of contamination of in-use MDVs in hospital settings. .-6 $^{-6}$ In these studies, none of 1,908 MDVs cultured was positive. Therefore, we can be $95 \%$ confident that the actual chance of contamination was no more than $0.2 \% .^{7}$

A laboratory study to characterize the effects of microbial contamination found marked differences in the growth supporting properties of eight different medications for single strains of 13 potential pathogens. ${ }^{8}$ The presence or absence of antimicrobial preservatives in the medications did not correlate with microbial survival. A hospital study of the usage patterns of MDVs indicated that the cost per dose from MDVs may be greater than expected because of unused and wasted medication. ${ }^{5}$ However, 28 of 50 medications in that study were only available in MDVs. In our hospital, the period which opened MDVs were available for use varied markedly between nursing units and between specific products. ${ }^{4}$ Many physicians insisted on opening new vials for each patient and each injection. In some areas, such as intensive care units and operating rooms, a discard-after-use policy was already in effect.

On the basis of existing information, then, the potential hazard posed by MDVs appears to be of a low order of magnitude. Expensive or frequently used medications probably need not be discarded until the expiration date. Infrequently used MDVs, such as those on resuscitation carts, that are entered hastily without due attention to asepsis should be discarded after a single use. Increased surveillance and documentation of infections are needed, and special attention should be directed to MDVs, such as those containing insulin or lidocaine, that tend to be in use for prolonged periods.

\section{REFERENCES}

1. Centers for Disease Control: Group A streptococcal abscesses after DTP immunization-Georgia. Morbidity Mortality Weekly Report 1982; 31:519-526.

2. Simmons BP, Hooton TM, Wong ES, et al: Guidelines for prevention of intravascular infections. Infect Control 1982; 3:61-72. 